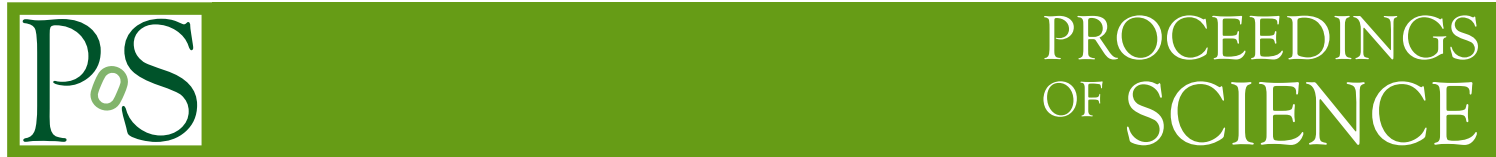

\title{
Cosmic-Ray Ionization of Molecular Clouds
}

\section{Nick Indriolo*}

Department of Astronomy, University of Michigan, Ann Arbor, MI 48109, USA

E-mail: indriolodumich.edu

Cosmic rays play a vital role in initiating the chemistry that occurs in molecular clouds. The ionization of $\mathrm{H}$ and $\mathrm{H}_{2}$ begins a network of ion-molecule reactions responsible for generating many of the diatomic and small polyatomic molecules observed in the interstellar medium. A few such species- $\mathrm{HCO}^{+}, \mathrm{DCO}^{+}, \mathrm{OH}^{+}, \mathrm{H}_{2} \mathrm{O}^{+}$, and $\mathrm{H}_{3}^{+}$in particular-are formed and destroyed by rather simple processes, making them powerful probes of the cosmic-ray ionization rate. I will discuss the current status of observations of these molecular ions within our Galaxy, as well as the conclusions that can be drawn from this ever-growing sample.

Cosmic Rays and the InterStellar Medium - CRISM 2014,

24-27 June 2014

Montpellier, France

\footnotetext{
* Speaker.
} 


\section{Introduction}

The interstellar medium (ISM) is home to a rich variety of atomic and molecular species, and the gas-phase chemistry responsible for forming many of these molecular species is driven by fast ion-molecule reactions. Generation of the ions necessary in this scheme requires a source of ionization, and cosmic rays-which can traverse large columns of material without being stopped-are widely accepted as the dominant ionizing mechanism. Because the formation of various molecules and molecular ions is closely linked to the ionization of $\mathrm{H}$ and $\mathrm{H}_{2}$ by cosmic-rays, observations of such species can be used to infer the cosmic-ray ionization rate, $\zeta$.

The rate of ionization of any species by cosmic rays is controlled by the energy dependent ionization cross section and the cosmic-ray flux as a function of particle energy. Ionization cross sections for $\mathrm{H}$ and $\mathrm{H}_{2}$ increase with decreasing energy below about $1 \mathrm{GeV}[1,2]$, so lower-energy particles are more efficient at ionizing both species. While the cosmic-ray flux above about $1 \mathrm{GeV}$ can be directly measured by experiments in Earth orbit, at lower energies the magnetic field coupled to the solar wind excludes such particles from the inner solar system. At present there is only one direct measurement of the local interstellar cosmic-ray spectrum down to about $2 \mathrm{MeV}$ [3]thanks to Voyager passing beyond the solar wind termination shock-and it is likely that this does not represent the low-energy particle flux throughout the entire Galaxy. Determining the cosmicray ionization rate via observations of various molecules can thus place useful constraints on the low-energy particle spectrum.

\section{Interstellar Chemistry}

Conditions within the ISM such as density $(n)$, kinetic temperature $(T)$, and radiation field vary widely between different environments, and determine what species will be present and how they form. Dense molecular clouds are cold $(T \leq 30 \mathrm{~K})$ and, by definition, dense $\left(n \geq 10^{4} \mathrm{~cm}^{-3}\right)$. They are opaque to optical and UV photons, which limits photodissociation processes and photoionizaion of metals such as $\mathrm{C}, \mathrm{S}$, and Si that are thought to be the primary sources of free electrons. This scarcity of electrons and energetic photons removes two of the main mechanisms by which molecules are destroyed, enabling the formation of more complex species. However, the low kinetic temperature inhibits reactions that must overcome potential energy barriers, blocking some routes forward in the chemical network. Clearly, some balance of these effects-and others-will determine the abundances of various atoms and molecules in dense clouds.

Diffuse molecular clouds have lower densities $\left(n \sim 100 \mathrm{~cm}^{-3}\right)$, warmer temperatures $(T \sim$ $70 \mathrm{~K}$ ), and are transparent to many optical/UV photons, which keeps metals singly ionized (i.e., $\left.\mathrm{C}^{+}, \mathrm{S}^{+}, \mathrm{Si}^{+}\right)$and the electron abundance high. Hydrogen is still predominantly in molecular $\left(\mathrm{H}_{2}\right)$ rather than atomic $(\mathrm{H})$ form, so that cosmic-ray ionization of $\mathrm{H}_{2}$ initiates the ion-neutral reaction network. In slightly warmer $(T \sim 100 \mathrm{~K})$, more tenuous $\left(n \sim 10-30 \mathrm{~cm}^{-3}\right)$ gas, hydrogen remains mostly in atomic form so that ionization of $\mathrm{H}$ is more important.

\subsection{Hydrogen Chemistry}

To demonstrate how observations of molecular species can be used in tracing the cosmic-ray ionization rate, consider the chemistry surrounding hydrogen in diffuse molecular clouds. $\mathrm{H}_{2}$ is 
first ionized by a cosmic ray,

$$
\mathrm{H}_{2}+\mathrm{CR} \rightarrow \mathrm{H}_{2}^{+}+e^{-}+\mathrm{CR}^{\prime},
$$

after which the $\mathrm{H}_{2}^{+}$ion reacts with $\mathrm{H}_{2}$,

$$
\mathrm{H}_{2}^{+}+\mathrm{H}_{2} \rightarrow \mathrm{H}_{3}^{+}+\mathrm{H}
$$

resulting in the formation of $\mathrm{H}_{3}^{+}$. Cosmic-ray ionization is the rate-limiting step in this process and can be taken as the formation rate of $\mathrm{H}_{3}^{+}$. In diffuse molecular clouds, $\mathrm{H}_{3}^{+}$is predominantly destroyed via dissociative recombination with electrons,

$$
\mathrm{H}_{3}^{+}+e^{-} \rightarrow \mathrm{H}_{2}+\mathrm{H} \text { or } \mathrm{H}+\mathrm{H}+\mathrm{H}
$$

and reactions (2.1)-(2.3) are an excellent approximation to the complete chemistry of $\mathrm{H}_{3}^{+}$. There are, of course, more reactions that can be considered, including

$$
\begin{gathered}
\mathrm{H}_{3}^{+}+\mathrm{CO} \rightarrow \mathrm{H}_{2}+\mathrm{HCO}^{+}, \\
\mathrm{H}_{3}^{+}+\mathrm{O} \rightarrow \mathrm{H}_{2}+\mathrm{OH}^{+},
\end{gathered}
$$

and

$$
\mathrm{H}_{3}^{+}+\mathrm{N}_{2} \rightarrow \mathrm{H}_{2}+\mathrm{HN}_{2}^{+},
$$

which account for other routes by which $\mathrm{H}_{3}^{+}$is destroyed, but the abundances of the reactants and the reaction rate coefficients mean these channels are negligible compared to dissociative recombination with electrons under typical conditions in diffuse molecular clouds.

The rate of change of the abundance of $\mathrm{H}_{3}^{+}$(or of any molecule) can be described as a differential equation that accounts for formation and destruction mechanisms, e.g.,

$$
\frac{d}{d t} n\left(\mathrm{H}_{3}^{+}\right)=n\left(\mathrm{H}_{2}^{+}\right) n\left(\mathrm{H}_{2}\right) k_{2.2}-n\left(\mathrm{H}_{3}^{+}\right) n(e) k_{2.3},
$$

where $n\left(\mathrm{H}_{2}^{+}\right) n\left(\mathrm{H}_{2}\right) k_{2.2}$ gives the formation rate of $\mathrm{H}_{3}^{+}, n\left(\mathrm{H}_{3}^{+}\right) n(e) k_{2.3}$ gives the destruction rate of $\mathrm{H}_{3}^{+}, n(X) \mathrm{s}$ are number densities of species $X$, and $k_{i} \mathrm{~s}$ are reaction rate coefficients for reaction $i$ herein. As stated above, reaction 2.1 is the rate-limiting step in $\mathrm{H}_{3}^{+}$formation, and so can replace the formation term in equation (2.7). Assuming steady state (i.e., constant $\mathrm{H}_{3}^{+}$abundance), the formation and destruction rates can be set equal to each other, giving,

$$
\zeta_{2} n\left(\mathrm{H}_{2}\right)=n\left(\mathrm{H}_{3}^{+}\right) n(e) k_{2.3},
$$

where $\zeta_{2}$ refers to the cosmic-ray ionization rate of $\mathrm{H}_{2}$. This can be re-arranged to solve for the ionization rate. Various substitutions (see [4] for detailed description) for more easily determined variables such as the electron fraction $\left(x(e) \equiv n(e) / n_{\mathrm{H}}\right.$, where $\left.n_{\mathrm{H}} \equiv n(\mathrm{H})+2 n\left(\mathrm{H}_{2}\right)\right)$ and for observable quantities such as column densities rather than number densities results in

$$
\zeta_{2}=k_{2.3} x_{e} n_{\mathrm{H}} \frac{N\left(\mathrm{H}_{3}^{+}\right)}{N\left(\mathrm{H}_{2}\right)} .
$$

Similar steady state analyses can be applied to other species (e.g., $\mathrm{OH}, \mathrm{OH}^{+}, \mathrm{HCO}^{+}$), and the interested reader is referred to a recent tutorial review article [5] for a more lengthy discussion on the chemical networks involved. 


\section{The Cosmic-Ray Ionization Rate}

Estimates of the cosmic-ray ionization rate based on molecular abundances have been made for over 40 years now. Dense cloud ionization rates are generally on the order of a few times $10^{-17} \mathrm{~s}^{-1}$, while diffuse cloud ionization rates are more typically a few times $10^{-16} \mathrm{~s}^{-1}$ [6]. This difference can be explained by the column density of material through which cosmic rays must travel to reach the two different environments. The low-energy particles most efficient at ionization will lose all of their energy before reaching the interiors of dense clouds, so that such environments will only be influenced by higher energy particles. This leads to a lower ionization rate in dense clouds than in regions such as diffuse clouds that low-energy particles still affect.

\subsection{Diffuse Clouds}

Many of the earliest estimates of the cosmic-ray ionization rate in diffuse clouds utilized observations of $\mathrm{OH}$ and $\mathrm{HD}[7,8]$. More recently, $\mathrm{H}_{3}^{+}$has become regarded as the most reliable tracer of the ionization rate as its formation and destruction mechanisms are rather straightforward (see above). Surveys searching for $\mathrm{H}_{3}^{+}$in diffuse molecular clouds now cover over 60 sight lines, and about half of those show $\mathrm{H}_{3}^{+}$absorption lines [4, 9]. The distribution of cosmic-ray ionization rates inferred from this sample of $\mathrm{H}_{3}^{+}$observations is roughly log-normal with mean $\log \left(\zeta_{2}\right)=-15.36$ $\left(\zeta_{2}=4.3 \times 10^{-16} \mathrm{~s}^{-1}\right)$ and standard deviation 0.24 , and is shown as a histogram in the top panel of Figure 1.

While $\mathrm{H}_{3}^{+}$observations are considered to be the most accurate means of inferring the cosmicray ionization rate, they are limited in scope due to the relative weakness of the absorption features (typically only 1-2\% deep), and the necessity for background sources bright at $3.7 \mu \mathrm{m}$ that are free of interfering absorption features. The Herschel Space Observatory has provided another means of estimating $\zeta$ by observing the related species $\mathrm{OH}^{+}$and $\mathrm{H}_{2} \mathrm{O}^{+}$, which form following cosmic-ray ionization of atomic hydrogen and charge transfer to oxygen. These particular Herschel observations use bright sub-mm continuum sources that can be several kpc distant as targets, probing all foreground gas in multiple intervening clouds via absorption spectroscopy [10, 11].

A survey targeting $\mathrm{OH}^{+}$and $\mathrm{H}_{2} \mathrm{O}^{+}$absorption in 20 sight lines throughout the Galaxy has detected both molecules in over 100 different velocity components [12]. Cosmic-ray ionization rates have been calculated in many of these components, and the resulting distribution is very similar to that found via $\mathrm{H}_{3}^{+}$(see bottom panel of Figure 1). Again the distribution is log-normal, and it has a mean value of $\log \left(\zeta_{2}\right)=-15.56\left(\zeta_{2}=2.7 \times 10^{-16} \mathrm{~s}^{-1}\right)$ and standard deviation 0.29. Combining both data sets, it is apparent that the cosmic-ray ionization rate in diffuse clouds has an average value of about $3 \times 10^{-16} \mathrm{~s}^{-1}$, but can vary between about $10^{-15} \mathrm{~s}^{-1}$ and several times $10^{-17} \mathrm{~s}^{-1}$.

\subsection{Dense Clouds}

Unlike diffuse cloud observations where molecules are seen in absorption toward some background continuum source, dense cloud observations rely on molecular emission lines. While the analysis of emission lines is generally more complex than that of absorption lines, the ability to map molecular emission removes the need for a bright background source, enabling observations over larger portions of the sky. 


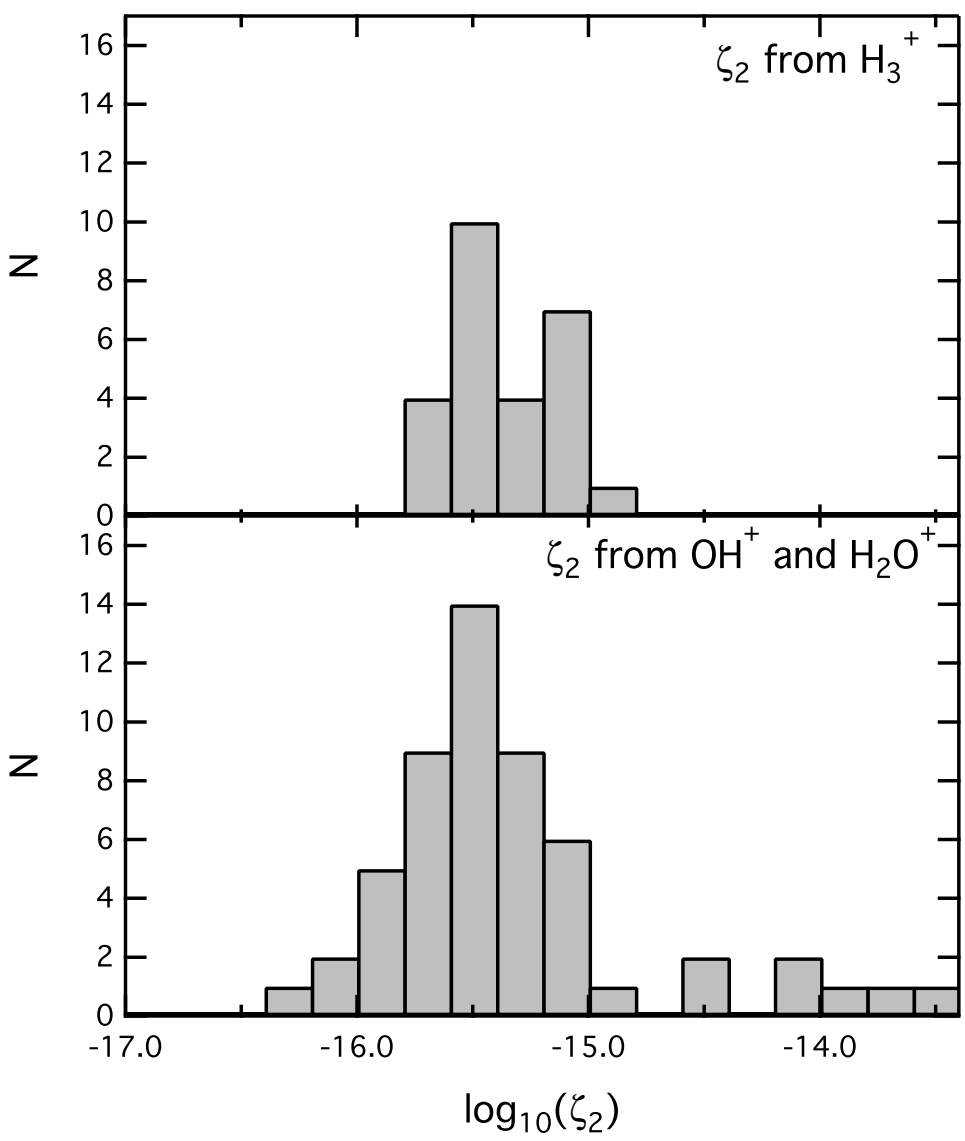

Figure 1: Reproduced from [12]. Histograms showing the distribution of cosmic-ray ionization rates determined from observations of $\mathrm{H}_{3}^{+}$(top panel) and from $\mathrm{OH}^{+}$and $\mathrm{H}_{2} \mathrm{O}^{+}$(bottom panel). All ionization rates above $\log \left(\zeta_{2}\right)=-14.8$ are inferred from gas in the Galactic center, and are not included in the distribution analysis.

Chemistry in dense clouds tends to be more complex than in diffuse clouds, with more reaction pathways leading to more species and larger species. For example, the lower electron abundance in dense clouds means that reactions (2.4)-(2.6) become important destructions mechanisms for $\mathrm{H}_{3}^{+}$. Beyond leading to more species though, this complexity also makes analytical approximations highly inadequate for describing molecular abundances. Instead, large systems of coupled differential equations accounting for hundreds of species and thousands of reactions are often utilized (e.g., [13]). Many times, simplified versions of these chemical networks (i.e., focusing only on a subset of related species and reactions) are also employed.

One group of molecules that has been used to infer the cosmic-ray ionization rate in dense clouds includes $\mathrm{HCO}^{+}, \mathrm{DCO}^{+}$, and $\mathrm{CO}$ [14]. These molecules are employed because the two ions are formed through reactions of $\mathrm{CO}$ with $\mathrm{H}_{3}^{+}$and with its deuterated isotopologues (e.g., $\mathrm{H}_{2} \mathrm{D}^{+}$), and so linked to $\zeta_{2}$. Ionization rates inferred from this analysis range from about $10^{-18} \mathrm{~s}^{-1}$ to $10^{-16} \mathrm{~s}^{-1}$ [14], but results are highly dependent on the assumed CO depletion factor (amount of $\mathrm{CO}$ frozen onto dust grains). More recent studies have attempted to account for these uncertainties 
by including physical models (i.e., temperature, density, and depletion profiles) within the chemical networks. Average ionization rates determined by such studies are a few times $10^{-17} \mathrm{~s}^{-1}$ [15]. These are in good agreement with earlier findings based on observations of $\mathrm{H}^{13} \mathrm{CO}^{+}$and physical models of the dense envelopes surrounding massive protostars [16].

\subsection{The Atomic ISM}

A recently detected molecule with the potential to constrain the cosmic-ray ionization rate in regions of almost purely atomic gas is $\mathrm{ArH}^{+}$[17]. This noble gas bearing molecule is formed via the ionization of $\mathrm{Ar}$ by cosmic rays, and the reaction of $\mathrm{Ar}^{+}$with $\mathrm{H}_{2}$. However, $\mathrm{ArH}^{+}$is also destroyed by reactions with $\mathrm{H}_{2}$, so too much molecular hydrogen will reduce the abundance below detectable limits. For this reason, we can be certain that $\mathrm{ArH}^{+}$is tracing low density, mostly atomic $\left(\mathrm{H}_{2} / \mathrm{H} \sim 10^{-4}\right)$ gas. Although not yet used for inferring $\zeta$, the formation process of $\mathrm{ArH}^{+}$clearly makes it a potential probe of the cosmic-ray ionization rate in environments not traced by any other species.

\section{Summary}

Observations of various molecules are used to infer the cosmic-ray ionization rate in different environments. In dense clouds $\mathrm{HCO}^{+}, \mathrm{DCO}^{+}$, and $\mathrm{CO}$ are used in tandem with both physical models and complex chemical networks, and average ionization rates are on the order of a few times $10^{-17} \mathrm{~s}^{-1}$. In diffuse clouds $\mathrm{H}_{3}^{+}, \mathrm{OH}^{+}$, and $\mathrm{H}_{2} \mathrm{O}^{+}$are observed in absorption, and independent analyses of $\mathrm{H}_{3}^{+}$and of $\mathrm{OH}^{+}$and $\mathrm{H}_{2} \mathrm{O}^{+}$give average ionization rates of a few times $10^{-16} \mathrm{~s}^{-1}$. The atomic ISM has now been probed via $\mathrm{ArH}^{+}$, and it is only a matter of time before this species is also used to infer $\zeta$. Although there are large variations in the ionization rates calculated in each environment (about 1-2 orders of magnitude in each case) the average diffuse cloud ionization rate is clearly about a factor of 10 larger than the average dense cloud ionization rate. This stems from the inability of low-energy cosmic rays - those most efficient at ionization-to reach the interiors of dense clouds, as all of their energy is used up ionizing gas in the outer layers of the clouds. It will be interesting to see if the same pattern holds true for regions probed by $\mathrm{ArH}^{+}$versus diffuse clouds, which would indicate a population of even lower energy particles incapable of penetrating diffuse clouds. By inferring ionization rates in all of these different environments, we can hope to determine the underlying cosmic-ray spectrum in each case. In doing so, we will learn more about how the particle spectrum changes with environment, and what this means about particle propagation through atomic and molecular gas.

\section{References}

[1] H. Bethe, Handbuch der Physik, Springer, Berlin 1933.

[2] M. Padovani, D. Galli, \& A. E. Glassgold, Cosmic-ray ionization of molecular clouds, A\&A 501619.

[3] E. C. Stone, A. C. Cummings, F. B. McDonald, B. C. Heikkila, N. Lal, \& W. R. Webber, Voyager 1 Observes Low-Energy Galactic Cosmic Rays in a Region Depleted of Heliospheric Ions, Science 341 150. 
[4] N. Indriolo, \& B. J. McCall, Investigating the Cosmic-Ray Ionization Rate in the Galactic Diffuse Interstellar Medium through Observations of $\mathrm{H}_{3}^{+}$, ApJ 74591

[5] N. Indriolo, \& B. J. McCall, Cosmic-Ray Astrochemistry, Chem. Soc. Rev. 427763

[6] A. Dalgarno, The Galactic Cosmic-Ray Ionization Rate, Proc. Nat. Acad. Sci. 10312269

[7] J. H. Black \& A. Dalgarno, Models of interstellar clouds. I - The Zeta Ophiuchi cloud, ApJS 34405

[8] T. W. Hartquist, J. H. Black, \& A. Dalgarno, Cosmic ray ionization and the deuterium abundance, MNRAS 185643

[9] N. Indriolo, T. R. Geballe, T. Oka, \& B. J. McCall, $\mathrm{H}_{3}^{+}$in Diffuse Interstellar Clouds: A Tracer for the Cosmic-Ray Ionization Rate, ApJ 6711736

[10] M. Gerin, M. de Luca, J. H. Black, et al., Interstellar $\mathrm{OH}^{+}, \mathrm{H}_{2} \mathrm{O}^{+}$and $\mathrm{H}_{3} \mathrm{O}^{+}$along the sight-line to G10.6-0.4, A\&A 518 L110

[11] D. A. Neufeld, J. R. Goicoechea, P. Sonnentrucker, et al., Herschel/HIFI observations of interstellar $\mathrm{OH}^{+}$and $\mathrm{H}_{2} \mathrm{O}^{+}$towards W49N: a probe of diffuse clouds with a small molecular fraction, A\&A 521 L10

[12] N. Indriolo, D. A. Neufeld, M. Gerin, et al., Herschel Survey of Galactic $\mathrm{OH}^{+}, \mathrm{H}_{2} \mathrm{O}^{+}$and $\mathrm{H}_{3} \mathrm{O}^{+}$: Probing the Molecular Hydrogen Fraction and Cosmic-Ray Ionization Rate, submitted to ApJ

[13] D. McElroy, C. Walsh, A. J. Markwick, M. A. Cordiner, K. Smith, \& T. J. Millar, The UMIST database for astrochemistry 2012, A\&A $\mathbf{5 5 0} 36$

[14] P. Caselli, C. M. Walmsley, R. Terzieva, \& E. Herbst, The Ionization Fraction in Dense Cloud Cores, ApJ 499234

[15] S. Maret, E. A. Bergin, \& M. Tafalla, Chemical modeling of the L1498 and L1517B prestellar cores: $\mathrm{CO}$ and $\mathrm{HCO}^{+}$depletion, $A \& A 559$ A53

[16] F. F. S. van der Tak \& E. F. van Dishoeck, Limits on the cosmic-ray ionization rate toward massive young stars, A\&A $358 \mathrm{~L} 79$

[17] P. Schilke, D. A. Neufeld, H. S. P Müller, et al., Ubiquitous argonium (ArH ${ }^{+}$) in the diffuse interstellar medium: A molecular tracer of almost purely atomic gas, A\&A 566 A29 